https://doi.org/10.18778/2196-8403.2021.05

BASTIAN LASSE

\title{
STEN NADOLNY und die Kunst der Lebensbeschreibung
}

Im Autobiographiediskurs spielen Romane bisher keine nennenswerte Rolle als theoretische Impulsgeber. Der vorliegende Beitrag zeigt, dass STEN NADOLNYs Selim oder Die Gabe der Rede eine autofiktionale Situation im inneren der diegetischen Welt erzeugt und sich damit für die Reflexion autobiographietheoretischer Problemkontexte eignet. Mit Rekurs auf Kristevas Überlegungen zur Selbst-Fremdheit werden die ,autofiktionalen' Schreibakte vorgeführt, die das Individuum auch vom Fremden her erschreiben. Als dezentrierte Selbstlebensbeschreibung überschreitet NADOLNYS Roman damit gängige Formen der Autofiktion.

Schlüsselwörter: Sten Nadolny, Selim oder Die Gabe der Rede, Autofiktion, Autobiographietheorie, Identität, Alterität

\section{Sten NAdOLNY and the art of life writing}

Discourse concerning autobiography does not usually include novels as material for theoretical study. This article demonstrates how STEN NADOLNY's novel Selim oder Die Gabe der Rede creates an autofictional situation within a diegetic world; therefore it may be a subject of reflection on theoretical problems associated with autobiography. In the context of Julia Kristeva's deliberations on self-alienation, the Author discusses 'autofictional' acts of writing that describe the self through the 'other'. As decentered life-writing, Nadolny's novel transcends common forms of autofiction.

Keywords: Sten NAdolny, Selim oder Die Gabe der Rede, autofiction, theory of autobiography, identity, alterity

\section{STEN NADOLNY i sztuka opowiadania życia}

W rozważaniach nad autobiografią gatunek powieściowy odgrywał do niedawna mało istotną rolę jako przedmiot badań. Artykuł pokazuje, że powieść STENA NADOLNEGO Selim oder Die Gabe der Rede tworzy w świecie przedstawionym sytuację autofikcjonalną. Tym samym można ten utwór poddać teoretycznej refleksji w odniesieniu do auto- 
biografii. W kontekście rozważań Julii Kristevy na temat obcości podmiotu wobec samego siebie, przedstawione zostają autofikcjonalne strategie pisarskie, które wywodzą autobiografię od ,obcego'. Powieść NADOLNEGO - można ją nazwać ,zdezorientowanym' opisem samego siebie - przekracza wobec tego tradycyjne formy autofikcji.

Stowa klucze: StEn NADOLNY, Selim oder Die Gabe der Rede, autofikcja, teoria autobiografii, tożsamość, bycie innym

\section{1. „Ich glaube an die Biographie!“}

Die Autobiographie ist in der Postmoderne in einer Krise, weil die „,biographische Illusion“ (BOURDIEU 1990:76) nicht mehr für ein außersprachliches Subjekt bürgen kann und dem „Ich“ mit dem „Ende jeglicher totalisierenden Synthesen“ (GUMBRECHT 2007:138) die Grundlage entzogen wird. Doch diese Konstellation eröffnet der Selbstlebensbeschreibung auch neue Perspektiven. In der aktuellen Diskussion wird die Fiktion als der Ausgangspunkt eines Lebens gesehen, das nicht in der Schrift beschrieben, sondern erschrieben wird (vgl. WAGNER-EGELHAAF 2006:357-358). Unter dem Stichwort der Autofiktion wird die Grenze zwischen fiktionaler und autobiographischer Darstellung für irrelevant erklärt, womit die Lizenz zur vollständigen Fiktionalisierung des eigenen Lebens ausgestellt werden kann. Insofern bleibt die autobiographische Lesart von Texten zwar nur noch eine Option (vgl. WAGNER-EGELHAAF 2006:368), bildet aber trotzdem eine „Bastion referentiellen Schreibens“ (HOLDENRIED 2017:57). Das hier in der Überschrift aufgenommene Zitat aus STEN NADOLNYS Roman Selim oder Die Gabe der Rede zeigt, dass nicht nur die Autobiographik an die postpostmoderne Möglichkeit der Selbstlebensbeschreibung, glaubt‘. Im Zentrum von NADOLNYS Romanen steht stets ein starkes Individuum (vgl. BOHNENKAMP 1996:36). So schreibt etwa Petra Günther: „Nadolnys Erzählweise hat nicht teil an der postmodernen Dissoziierung des Ich“ (GÜNTHER 1993:44). NADOLNY praktiziere ein „Erzählen gegen den Tod des Individuums“ (BOHNENKAMP 1996:37). Dieses Individuum kommt im Roman Selim sogar zu seinem Recht, eine Selbstlebensbeschreibung zu liefern.

Der Protagonist des Romans, Alexander, begegnet den Leser*innen auf mehreren Erzählebenen und erzählt seine Geschichte in der Form eines ,Romans ${ }^{1}{ }^{1}$ und graphisch abgesetzten Tagebuchpassagen. Der erzählte Zeitraum erstreckt sich vom Jahr 1965 bis zum Jahr 1989 und streift dabei wichtige Ereignisse der

$1 \quad$ Im Folgenden bezeichnet ,Roman“ in einfachen Anführungszeichen Alexanders Roman innerhalb von NADOLNYS Text. 
deutschen Geschichte wie die einsetzende Arbeitsmigration, die Studentenbewegung und den deutschen Herbst. Der ,Roman' im Roman behandelt dabei auch die Lebensgeschichten mehrerer Weggefährten Alexanders, darunter die des jungen türkischen Arbeitsmigranten Selim. Alexanders Entwicklung vom Wehrdienstleistenden, Studierenden und Rhetoriklehrenden zum Millionär wird verknüpft mit Selims Werdegang. Selim, der die titelgebende „Gabe der Rede“ besitzt und Alexander als begnadeter Geschichtenerzähler erscheint, verstirbt im Romanverlauf. Alexander entwickelt sich selbst zum Erzähler und nimmt damit das ,Erbe' Selims an.

Der Roman konstruiert durch seine Gegenüberstellung von ,Roman“ und Tagebuch innerhalb der Fiktion eine fiktionale Ebene und eine Ebene, die den Status einer ,Realität' beansprucht. Die Lebensbeschreibung, die Alexander mittels des ,Romans' entwirft, steht demnach im gleichen theoretischen Problemkontext wie die Autofiktion, auch wenn keine Referenzbeziehung zu einer außertextuellen Ebene besteht. Weiterhin problematisiert der Roman innerhalb der Textwelt eindeutige Referenzbeziehungen zwischen den Ebenen Tagebuch und ,Roman“. Bereits im Prolog fragt Alexander: „Wer war dieser Selim, wer ist er, vor allem: wo?" (NADOLNY 2005:9). Anders könnte gefragt werden: Ist Selim überhaupt eine eigenständige Figur oder nicht vielmehr ein Alter Ego des Autors Alexander? Und ließe sich eine solche Konstellation nicht als produktive Antwort auf die Frage, wie nach der Krise der Autobiographie noch Selbstlebensbeschreibungen verfasst werden können, verstehen? Die vorliegende Studie will Selim unter diesen Gesichtspunkten lesen. NADOLNYS Roman, so die hier vertretene These, schreibt sich in den Autobiographie-Diskurs ein und macht ein Angebot, wie ausgehend von der Fiktion unter Ausklammerung eines außertextuellen bios eine Selbstlebensbeschreibung in Zeiten postmoderner Subjektkrisen aussehen könnte.

Die Arbeit argumentiert zunächst dafür, einen ,rein' fiktionalen Text wie Selim als theoretischen Beitrag in den autobiographischen Diskurs zu integrieren. Im Anschluss daran wird gezeigt, wie sich Selim an den autobiographischen Diskurs anschließen lässt. In einem nächsten Schritt erörtere ich, inwiefern Selim als Manifestation des Selbst-Fremden Alexanders betrachtet werden kann. Wenn anstelle des Ichs ein Anderer für die Selbstkonstruktion einsteht, dann wird die Autofiktion um einen entscheidenden Aspekt ergänzt, wenn nicht gar überschritten. Diese Leistung des Romans wird im Fazit eingehender beleuchtet. 


\section{Autofiktion}

Die theoretische Diskussion um die Autobiographie ist vor allem durch Philippe Lejeunes Konzept des autobiographischen Paktes geprägt. Ausgehend von der Annahme, dass der Eigenname die einzige Markierung im Text sei, die auf eine tatsächliche Person verweise und so die Textäußerung an ein außersprachliches Subjekt binde (vgl. LEJEUNE 1994:23), definiert Lejeune die Namensidentität von Autor, Erzähler und Protagonist als notwendiges Kriterium jeder Autobiographie (vgl. LEJEUNE 1994:27). Der autobiographische Pakt verbürgt diese Identität: „Der autobiographische Pakt ist die Behauptung dieser Identität im Text, die letztlich auf den Namen des Autors auf dem Umschlag verweist" (LEJEUNE 1994:27). Als Gegenstück zum autobiographischen Pakt bestimmt Lejeune den Romanpakt. Dieser werde immer dann geschlossen, wenn paratextuelle Elemente die Fiktionalität des Textes bestätigten und keine Namensidentität zwischen Autor, Erzähler und Figur bestehe (vgl. LEJEUNE 1994:29). Diesen beiden Pakten stellt Lejeune den Referenzpakt zur Seite, der eine Beziehung zwischen Text und Realität garantiere (vgl. LEJEUNE 1994:40).

Parallel zu Lejeunes folgenreichem Einwurf in die Theoriedebatte verändern sich seit den 1960er Jahren sowohl die autobiographische Produktion als auch ihre wissenschaftliche Rezeption entscheidend. Die hermeneutische Gleichsetzung von Lebens- und Sinnzusammenhang wird aufgehoben und der Zweifel an der Verfügbarkeit eines Erzählobjekts, des autobiographischen Ichs, macht den behaupteten Zusammenhang von Leben und Text zu einer Referenzillusion (vgl. HOLDENRIED 2000:45). Die Abwendung von einer hermeneutischen Konzeption der Autobiographie verändert den Impetus der Gattung: „Autobiographie ist in dieser Sicht keine definierte Gattung der Lebensdarstellung mehr, sondern ein Problem der Schrift (,graphie'), deren Selbstrückbezüglichkeit (,auto') ein Eigenleben (,bios') hervorbringt" (WAGNER-EGELHAAF 2005:80). Diese Fiktionalisierung gewährleistet die vornehmliche Gattungsfunktion, die Konstruktion von Subjektivität (vgl. HOLDENRIED 2017:58). Über die Fiktion soll nicht mehr ein einheitliches Subjekt zur Synthese gebracht, sondern die ,Wirklichkeit' eines seit der Postmoderne fragmentierten Ichs konstituiert werden (vgl. WAGNER-EGELHAAF 2010:197).

Als Stichwort hat sich dazu der im Jahr 1977 von Serge Doubrovsky geprägte Begriff der Autofiktion etabliert, dessen Konzeptualisierung im Folgenden aus der Perspektive der deutschen Theoriebildung skizziert wird. ${ }^{2}$ In einer weiten

Für eine Perspektive auf die französische Theoriebildung siehe BENNE 2007. 
Definition wird davon ausgegangen, dass eine Autofiktion immer dann vorliegt, wenn zwischen dem Autor und einer Figur Namensidentität besteht, aber durch paratextuelle Faktoren ein Romanpakt angeboten wird (vgl. ZIPFEL 2009:302). Die autobiographische Lesart wird im autofiktionalen Verständnis zu einer möglichen, aber nicht zwingenden Option, da die Alternative von Wirklichkeit oder Fiktion im Gegensatz zur Möglichkeit einer produktiven Selbsterzeugung mittels der Sprache keine entscheidende Rolle spielt (vgl. WAGNER-EGELHAAF 2006:361). Im engeren Sinn bezeichnet Autofiktion die Verschränkung von Fiktion und Wirklichkeit (vgl. WAGNER-EGELHAAF 2016:19), eine Verbindung, die sowohl in der klassischen Autobiographie als auch im autobiographischen Roman auffindbar ist (vgl. WAGNER-EGELHAAF 2016:21). Diese Überschneidungen in den Begriffen führen dazu, dass Autofiktion mitunter nicht als eigenständiger Begriff verwendet wird und unter die Kategorie des autobiographischen Romans subsumiert wird (vgl. HOLDENRIED 2017:57). Die Eigenständigkeit der Begriffsbildung erklärt Martina Wagner-Egelhaaf wie folgt: ,[D]er Begriff ist aber genau dann und deshalb brauchbar, wenn es um die Beschreibung von Projekten geht, die ihre Ästhetik auf das gezielt eingesetzte Wissen von der Fiktionalität des Faktischen und der Wirklichkeitsmacht der Fiktion abstellen" (WAGNER-EGELHAAF 2016:21).

Eine Typologie autofiktionalen Erzählens entwickelt Frank Zipfel (vgl. ZIPFEL 2009). Der erste Typ autofiktionalen Schreibens zeichne sich dadurch aus, dass eine ,eigentliche" Autobiographie mit dem Romanpakt versehen werde. Unter dem Eindruck einer nicht mehr konkreten Referenz werde der Referenzpakt nur noch unter Vorbehalt akzeptiert (vgl. ZIPFEL 2009:301). Der zweite Typ sei als eine besondere Art des fiktionalen Erzählens zu klassifizieren. Unter Rückgriff auf Gérard Genette wird die Autofiktion in den Bereich der Fiktion eingeordnet und aus dem Bereich autobiographischen Schrifttums ausgenommen (vgl. ZIPFEL 2009:302). In einem dritten Typ versteht Zipfel als Autofiktion die Kombination von autobiographischem Pakt und Romanpakt. Der Text sei in diesem Fall weder eindeutig als autobiographisch noch als fiktional zu bestimmen, sodass die Rezeptionshaltung zwischen den Pakten oszilliere (vgl. ZIPFEL 2009:305). Alle möglichen Formen autofiktionalen Schreibens lassen sich im Kern auf eine Kritik an traditionellen autobiographischen Konzepten zurückführen und schließen demnach an die antihermeneutische Wende in der Autobiographik an. Im Sinne der postmodernen Kritik wird die autobiographische Praxis unter dem Vorzeichen der Autofiktion ,neu' gedacht und als Konstruktionsprozess des Selbst aufgefasst (vgl. ZIPFEL 2009:307-308). Trotz der starken Präsenz des Begriffs der Fiktion im Diskurs um die Selbstlebensbeschreibung wird die 
theoretische Debatte immer noch vom Versuch getragen, Leben und Schrift zu verbinden. Wie der gesamte autobiographische Diskurs weist auch die Autofiktionsdebatte die Tendenz auf, trotz der Referenzproblematik die Autobiographik an ein außersprachliches Subjekt rückzubinden. Die Möglichkeit, eine Selbstlebensbeschreibung ohne ein tatsächliches Leben zu denken, scheint aus der Debatte ausgeschlossen zu sein. So zeigt Zipfels zweiter Typ der Autofiktion, dass ein einmal in den Bereich der Fiktion eingeordneter Text aus dem engen Bereich der Autobiographik ausgeschlossen wird und somit auch einen Status verliert, der autorisierte Aussagen in Bezug auf dieses Diskursfeld machen kann. Autofiktion heißt nach Wagner-Egelhaaf zwar, „sich nicht mehr an der Grenze abarbeiten zu müssen, die vermeintlich zwischen fiktionaler und autobiographischer Darstellung besteht" (WAGNER-EGELHAAF 2006:368), aber die daraus gezogene Konsequenz, nach der ein bios durch graphie hervorgebracht werden könne (vgl. WAGNER-EGELHAAF 2006:368), zeigt, dass die Diskussion nach wie vor an dieser Grenze angesiedelt ist. Wenn wir der „Wirklichkeitsmacht der Fiktion“ (WAGNER-EGELHAAF 2016:21) zu vollem Recht verhelfen wollen, darf die außertextuelle Referenz nicht mehr das Einschlusskriterium für Diskursrelevanz darstellen. Darum hier mein Plädoyer dafür, Romanprojekte auf ihr kritisches Interventionspotential hin zu befragen. Es geht mir im Folgenden also nicht darum, Selim als Autofiktion zu lesen, die einen außertextuellen bios hervorbringt. Stattdessen will ich herausstellen, welche an die Debatte der Selbstlebensbeschreibung adressierten Vorschläge sich über die Lektüre dieses Texts generieren lassen.

\section{Selim als Autofiktion}

Die ergiebigste Quelle einer Reihe von kritischen Aussagen zum autobiographischen Diskurs findet sich im zwölften Kapitel des Romans, das überwiegend aus „Tonbandnotizen“ besteht, die sich mit dem Zusammenhang von Erzählen und Ökonomie befassen. Hierbei entstehet eine Poetologie, die zunächst das Gewichtungsverhältnis von Leben und Geschichte umkehrt: „Wer entschieden hat, welche Geschichte er mit seinem Leben erzählen will, lebt möglicherweise folgerichtiger, aber wenn er sich irrt, tut er auch das gründlicher [...]" (NADOLNY 2005:407-408). Das Leben wird hier nicht nur als etwas begriffen, das in einer Geschichte dargestellt werden kann. Darüber hinaus wird das Leben zum Rohmaterial einer narrativen Form, die mit eigenem Recht Bestand hat. Somit verknüpft sich diese Aussage mit der autofiktionalen Theorie insofern, als auch diese betont, wie durchlässig die Grenzen zwischen Erzählen und Leben letzt- 
lich sind. Im Anschluss an diese Erkenntnis problematisiert die Autofiktion das Verständnis von ,Wahrheit' und stellt heraus, wie die Fiktion ,Wirklichkeit hervorbringen kann.

Selim thematisiert dieses Problem der Wahrheit im Kontext eines postmodernen Zweifels an den teleologischen ,Meistererzählungen' und knüpft an die Kritik der am hermeneutischen Modell geschulten autobiographischen Form an:

Wer der Wahrheit sklavisch dient, liebt sie nicht. Selim weiß, daß sie ungern persönlich in Erscheinung tritt, sondern es vorzieht, sich von Abertausenden von Geschichten annäherungsweise nachbilden zu lassen. (NADOLNY 2005:418)

Die „Abertausenden von Geschichten“ ersetzen die eine Erzählung und die ,Wahrheit' wird zu einem nicht fixierbaren Kern, der sich lediglich einkreisen lässt. Trotz der Kritik an der einen, zusammenhängenden Form erscheint hier ein hermeneutisches Erkenntnismodell weiterhin intakt, das ebenfalls nicht davon ausgeht, dass ein abschließendes Erkennen möglich sei. Die ,uneigentliche“ Erscheinung der Wahrheit in „Abertausenden von Geschichten“ kann sogar an ein hermeneutisches Autobiographiekonzept angebunden werden. Goethe postuliert in Dichtung und Wahrheit, dass gerade die Dichtung die Wahrheit hervorbringe (vgl. WAGNER-EGELHAAF 2006:354). So besehen ,versteckt' sich hinter den „Abertausenden von Geschichten“ die goethesche Dichtung als Mittlerin der Wahrheit. Goethes Konzept wird von Martina Wagner-Egelhaaf als autofiktionales Schreiben gedeutet (vgl. WAGNER-EGELHAAF 2017:15). Der hier hergestellte Bezug auf Goethe kann einerseits als Anschluss an die Autofiktion, andererseits aber auch als Aktualisierung eines hermeneutischen Modells verstanden werden.

Durch das Erzählen wird nur das Individuum sicher. Diese Quintessenz beschließt die poetologischen Ausführungen Alexanders: „Das Erzählen trägt uns wie die See den Seemann: nichts wird durch sie sicherer, nur er selbst" (NADOLNY 2005:419). ,Sicher' wird das Individuum dadurch, dass es sich in der Schrift nicht nur produziert, sondern auch fixiert. Es steht im Medium der Schrift sicher gegen die Umwelt, die im Bild der „See“ als unbeständig erscheint. Der Text wird zu einem Refugium für das Individuum: „,in der Schwundstufe einer erzählerischen Geste" (WAGNER-EgELHAAF 2006:366) wird das Subjekt im Text neu eingesetzt. Das Erzählen garantiert in Selim wie in der autofiktionalen Theorie das Fortbestehen der Subjektivität.

Der Status des Subjekts, das Verständnis von Wahrheit und nicht zuletzt das Verhältnis von ,Wirklichkeit' und ,Fiktion' werden in der textinternen Poetologie reflektiert. Alle Reflexionen bieten Anschlüsse an die Theoriebildung im Feld der Autobiographieforschung und zeigen in der Verbindung mit der 
Struktur des Textes, wie stark der Roman in dessen Diskurse ,verstrickt ${ }^{\star}$ werden kann. Der Roman bietet aber nicht nur auf der Ebene der histoire Anknüpfungspunkte an die Autofiktion. Es ist insbesondere der discours, der eine neuartige Figuration dieses Phänomens hervorbringt.

Im Roman gibt es zwei Erzählebenen, die zunächst asynchron ablaufen, jedoch im weiteren Verlauf ineinander fallen. Die erste Erzählebene lässt sich als konventionelle auktoriale Erzählung charakterisieren, die in einer ,alternierenden Parallelmontage“" (BUNZEL 1996:148) das zeitgleich ablaufende Geschehen um die verschiedenen Figuren an ihren jeweiligen Handlungsorten beschreibt (vgl. BUNZEL 1996:148-149). Graphisch durch kursive Schrift abgesetzt, besteht die zweite Erzählebene aus den Tagebuchaufzeichnungen Alexanders (vgl. BUNZEL 1996:149). Wolfgang Bunzel beschreibt die dadurch entstehende Konfiguration des Textes wie folgt: „Alexander und Selim sind daher nicht einfach erfundene Figuren, sondern sie beanspruchen - innerhalb der Fiktion von NADOLNYS Roman - Dignität als reale Personen“" (BUNZEL 1996:149).

Anders gesagt werden der autobiographische Pakt und der Referenzpakt ins Innere des literarischen Textes verschoben, wodurch der ,reale“ Autor der außertextuellen Ebene aus dem Blickpunkt des Interesses gerät. Als Autor des Tagebuchs verbürgt Alexander mit seinem Namen die ,Referenz' der extradiegetischen Ebene (Tagebuch) auf die intradiegetische Ebene (,Roman') und damit die Kongruenz der Ebenen und Figuren. Somit entsteht eine autobiographische Situation, in der die „Dignität als reale Personen“ erst entstehen kann. Der Name des Autors (Alexander) entspricht dem Namen einer Figur (Alexander) bei gleichzeitigem Angebot eines Romanpakts. Über die Verschränkung der zwei Textebenen erhält der Text gemäß Zipfels zweiter Definition demnach eine autofiktionale Struktur.

Im 117. Unterkapitel wird eine weitere typographisch abgehobene Textform eingeführt (vgl. NADOLNY 2005:283). Bunzel sieht in diesen formal eigenständigen Absätzen eine dritte Erzählebene und ein Bindeglied zwischen Tagebuch und ,Roman“ (vgl. BunZEL 1996:153). Das Tempus der Absätze ebenso wie die Datumsangaben schlügen eine formale Brücke zum Tagebuch, während die Erzählform und die Schriftart eine Anbindung an den Roman ermöglichten (vgl. BUNZEL 1996:153). In ihrer Abfolge führten die drei Textarten die Transposition vom Dokument zum Kunstgebilde vor (vgl. BUNZEL 1996:154). Ähnlich wandelt sich die Autobiographie, zugespitzt formuliert, vom Ego-Dokument, das unter historiographischen Gesichtspunkten gelesen wird, zur Autofiktion, in welcher der fiktionale Charakter funktionales Gewicht erhält. Die formale Gestaltung des Romans spiegelt diese Entwicklung und vollzieht sie als Text- 
transformation nach. Ferner zeigen die Übergänge die Vermischung von ,Fakt ${ }^{\star}$ und ,Fiktion' innerhalb der Textebenen, wodurch die Struktur des Romans auch hier Parallelen zur autofiktionalen Theoriebildung aufweist.

Die Vermischung von ,Fakt' und ,Fiktion“ innerhalb des literarischen Textes lässt sich weiterhin an der Verschränkung der Textebenen über die Einbindung von ,Versatzstücken' nachzeichnen. So finden sich einerseits ,Realien“ aus der extradiegetischen Ebene im ,Roman'. Beispielhaft ist der Entwurf für Alexanders Redekurse, der in den ,Roman' aufgenommen wird (vgl. NADOLNY 2005:334-336). Auf der anderen Textebene finden sich Abschnitte aus dem ,Roman', die ,an entsprechender Stelle“ (NADOLNY 2005:261) eingefügt werden sollen. Die Beziehung zwischen ,Roman'- und Tagebuchtext wird hier besonders enggeführt, da die Versatzstücke für den ,Roman“ informell durch das Tagebuch kommentiert werden. Exemplarisch steht hierfür Alexanders im Jahr 1968 gehaltene Rede.

Die Passage ist zunächst intern auf Alexander fokalisiert, eine Perspektivierung, die die Rede als großen Misserfolg erscheinen lässt. Durch die Auflösung der aktorialen Mitsicht wird diese Darstellung relativiert: „Sein Auftritt war so schnell wieder vergessen wie fast alles jetzt" (NADOLNY 2005:264-265). Alexanders Tagebuch kommentiert daraufhin: „Zu überlegen ist, ob ich erwähne, wie viele sich später doch noch daran erinnerten" (NADOLNY 2005:265). Der vermeintliche ,Fakt', der durch die aufgebrochene Perspektive am Ende des ,Roman'-Ausschnitts zunächst ,objektiv' wirkt, wird durch die extradiegetische Textebene relativiert, indem der Erzählerrede widersprochen und eine Einschätzung einer außenstehenden Person integriert wird: „Ein meisterhafter Abgang!“(NADOLNY 2005:265). Im Wechselspiel der Ebenen wird hier also der relative Status von ,Fakten' vorgeführt und der autofiktionale Charakter des ,Romans“ hervorgehoben. Das Spiel mit den Perspektiven zeigt dabei die möglichen Akzentuierungen des biographischen Ereignisses auf, ohne die dabei entstehenden Mehrdeutigkeiten kohärent aufzulösen.

Die Verschränkung der beiden primären Textebenen, die Einführung einer dritten Erzählebene, die eingefügte Binnenerzählung um die türkische Migrantin Ayşe (NADOLNY 2005:369) und das ,phantastische“ Kapitel „Selim siegt“ (NADOLNY 2005:476) machen die Fragmentierung des ,Romans' im Roman offensichtlich. Bunzel zeigt, dass das Erzählgeschehen in Bruchstücke zerfällt, sobald zwischen den beiden Fiktionsebenen keine zeitliche Distanz mehr besteht. Er argumentiert, dass mit der chronologischen Konvergenz der Fiktionsebenen der für den Schreibprozess notwendige Überblick verloren gegangen sei und der bisher kontinuierliche Romanfortgang dadurch gestört werde (vgl. BUNZEL 1996:153-154). Anders gesagt verschwindet mit dem zeitlichen Zusammenfall 
der Erzählebenen die Distanz zwischen erzählendem und erzähltem Ich. Das Kapitel „In der Türkei“ (NADOLNY 2005:428) macht deutlich, welche Auswirkungen diese Konvergenz auf das Verhältnis der Erzählebenen hat. Zunächst gibt die Tagebuchnotiz vom 11. August 1988 darüber Auskunft, dass die Reise in die Türkei von Beginn an als Projekt geplant ist, das Leben und Schreiben als parallel ablaufende Prozesse versteht: „In der Türkei werde ich zweierlei zur gleichen Zeit tun: erstens reisen und Selim finden, zweitens ebendies beschreiben [...]" (NADOLNY 2005:429). Wenn auch Leben und Schreiben synchron ablaufen, erscheint hier noch ein mimetisches Verhältnis von Schrift und Leben: das Beschreiben wird als sekundärer Prozess begriffen, der die Ereignisse der Reise abbildet. Dieses Verhältnis verändert sich, als Alexander vom Tod Selims erfährt und das ursprüngliche Projekt gescheitert ist: „Ich schreibe das um! Schreibend werde ich ihn finden, und dafür werde ich reisen [...]" (NADOLNY 2005:433). Die Erzählung wird hier zur Autofiktion, denn die Geschichte soll das Leben hervorbringen, nicht etwa abbilden. Die intradiegetische Ebene wird insofern ,ermächtigt', als sie nicht mehr als mimetisches Abbild der extradiegetischen Ebene präsentiert wird. Sie soll nach dem Willen des Autors Alexander nun die Realität sein, das erzählte Ich wird im Sinne einer Autofiktion ,lebendig“. Dementsprechend wird das Verhältnis der Erzählebenen radikal in Frage gestellt. Plötzlich ist es nicht mehr die extradiegetische Ebene des Tagebuchs, die den ,Roman' hervorbringt, sondern der Roman produziert das Tagebuch und seinen autodiegetischen Erzähler Alexander: ,Alexander saß auf der Terrasse unter dem Blätterdach und schrieb Tagebuch" (NADOLNY 2005:455). Direkt anschließend folgt die Tagebuchnotiz vom 27. August 1988, hier auszugsweise zitiert:

Alexanders verdammtes Fieber lasse ich ab sofort nicht mehr in den Roman! [...] Womöglich passiert ihm etwas, und ich überlebe ihn um Jahre. Aufhören mit dem Tagebuchschreiben, das kostet nur Energie! Weiter mit dem Roman, mag er noch so auf der Stelle treten [...]. (NADOLNY 2005:455)

Das Eigenleben, das die ,Roman'-Figur zu führen scheint, wird in der Dissoziation von Personalpronomen und Eigennamen deutlich. Das Ich des Tagebuchs und Alexander, die im Romanverlauf bisher aufeinander referiert haben, fallen auseinander. Die sich erschreibende Person erhält dabei sogar den Vorzug vor der vermeintlich, realen' Person: das Tagebuch, das dieser ,realen' Person eine Gestalt gibt, soll zugunsten des ,Romans' nicht mehr weitergeführt werden. Das Ich, bisher definiert vom Tagebuch, wird zunehmend vom ,Roman' beherrscht. „Ich soll also über diesem Roman sterben?“ (NADOLNY 2005:456). Diese in Alexanders Tagebuch gestellte Frage kann auch losgelöst von der immer wieder beschworenen Gefahr durch ein schweres Fieber gelesen und auf die ,Bedrohung' des Ich durch die Autofiktion bezogen werden. Die Autonomie des 
,Romans“ reflektiert das Tagebuch indes weitaus früher: „Während ich mir Gedanken mache und Distanz zu gewinnen suche, schreibt er sich selbst weiter, auto-biographisch, Nacht für Nacht. Er braucht mich nur aus zwei Gründen: Ich bin eine seiner Figuren, und - ich kann Maschine schreiben" (NADOLNY 2005:162). Das Eigenleben des ,Romans' und die ,autobiographische Kraft ${ }^{\text {‘ }}$ werden demnach früh realisiert. Diese Kraft erhält im Kapitel „In der Türkei“ darüber eine neue Dimension, dass sie selbst Materialität beansprucht und zur Autofiktion wird. Die Urheber-Verhältnisse zwischen erzähltem und erzählendem Ich werden an der Grenze zwischen ,Fakt‘ und ,Fiktion“ unklar. In diesem Sinne gibt es keine ,faktische‘ Ebene mehr und Alexander wird im Wechselspiel von Tagebuch und ,Roman` autofiktional erschrieben.

\section{Dezentrierte Selbstlebensbeschreibung}

„Ich fange an zu begreifen, was dich mit Selim verbindet. [...] Es handelt sich bei Alexander und Selim um ein und dieselbe Person! Alexander, das bist du als Unfertiger, Suchender, und Selim, das bist du als gestandener Mann" (NADOLNY 2005:321). Diese Interpretation des mit Alexander befreundeten Regisseurs Olaf begründet die Möglichkeit einer doppelten Referenz des Tagebuch-Ichs. Der autodiegetische Erzähler weist diese Interpretation entschieden zurück: „Ich antworte, Interpretationen wie diese seien so scharfsinnig, daß jede Geschichte sich vor ihnen in acht nehmen müsse“ (NADOLNY 2005:321). Der Ich-Erzähler restituiert die Eindeutigkeit der Referenz und unterstreicht die Abwegigkeit von Olafs Interpretation noch mit einem Verweis darauf, dieser „dürfte nicht allzu genau gelesen haben“ (NADOLNY 2005:321). Für die klassische Theorie der Autobiographie ist Olafs Interpretation problematisch, weil das Kriterium der Namensidentität verletzt wird. Für Lejeune kann keine Autobiographie vorliegen, wenn der Autor sich als Figur mit anderem Namen beschreibt:

Wenn eine Person, die ihr Leben schildert, einen fiktiven (also von dem des Autors verschiedenen) Namen erhält, so kommt es vor, daß der Leser Grund zur Vermutung hat, daß die von der Figur erlebte Geschichte genau die des Autors ist [...]. Hätte man auch die besten Gründe der Welt zur Vermutung, daß die Geschichte genau dieselbe ist, so ist der dergestalt hervorgebrachte Text dennoch keine Autobiographie [...]. (LEJEUNE 1994:26)

Olafs Interpretation beruht nicht auf dem für Lejeune notwendigen Kriterium der Identität, sondern auf der Kategorie der Ähnlichkeit. Damit wäre Selim ein autobiographischer Roman, in dem die Leser*innen ,aufgrund von Ähnlich- 
keiten [...] Grund zur Annahme ha[ben], daß eine Identität zwischen Autor und Protagonist besteht, während der Autor jedoch beschlossen hat, diese Identität zu leugnen oder zumindest nicht zu behaupten“" (LEJEUNE 1994:26). Eine auf der Kategorie der Ähnlichkeit beruhende Identitätsbeziehung zwischen dem Autor Alexander und der Figur Selim ist demnach prinzipiell möglich, schlösse den Text jedoch aus dem autobiographischen Diskurs aus.

Zumindest in der deutschen Theoriebildung geht die Autofiktion in diesem Punkt nicht wesentlich über Lejeunes Konzept hinaus. Wie Zipfels Typologie der Autofiktion zeigt, ist auch hier die Namensidentität konstitutionell für die Möglichkeit einer autobiographischen Lesart. Ein hier notwendiger Seitenblick auf die französische Theoriebildung zeigt die Ausnahme von der Regel auf. Jacques Lecarme schreibt mit Bezug auf den Autor: „,[O]n peut proposer au lecteur de décoder le nom du narrateur pour y lire celui de l'auteur" (LECARME 1993:236). Sebastian Brass nimmt diese Überlegungen zum Ausgangspunkt dafür, die durch ein Allonym aufgerufenen Spannungen zwischen Identität und Differenz als Inkubator des wesentlichen Moments autofiktionaler Darstellung zu verstehen, die er als das „Nach-außen-Setzen des ,Je est un autre““ bestimmt (BRASS 2021:74). Das von Olaf beschriebene Phänomen der doppelten Referenz kann demnach nur dann als autobiographietheoretisch relevant diskutiert werden, wenn man wie Brass vom Paktmodell abrückt. Olafs Interpretation bietet einen Ansatzpunkt, um ein Konzept einer Selbstlebensbeschreibung abzuleiten, welches das Selbst auch vom Fremden aus erschreibt und damit der Dissoziation des Ichs in der Postmoderne gerecht wird. Diese Doppelzentrierung auf zwei Fokusse, oder Dezentrierung des Selbst in der Selbstlebensbeschreibung, lässt sich paradigmatisch in Selim aufzeigen.

Bereits in einer der ersten Tagebuchnotizen stehen folgende Sätze: „Selim ist eine Geschichte, die ich erzählen will. Selim ist einer, dem ich verpflichtet bin" (NADOLNY 2005:47). Die Konstruktion fällt sowohl syntaktisch als auch durch ihre symmetrische graphische Anordnung im Roman als Parallelismus auf. Dadurch wird sprachlich und graphisch eine Gleichzeitigkeit von zwei Selims nahegelegt. Zum einen ist dieser Selim eine Geschichte, die das Ich des Tagebuchs erzählen will. Diese Geschichte hängt existentiell vom Erzähler ab, ist also referentiell auf ihn bezogen. Selim ist hier die Geschichte Alexanders. Gleichzeitig ist Selim aber als eigenständige Person auf der Tagebuchebene anwesend. Diesem Selim ist das Ich im Sinne einer biographischen Redlichkeit verpflichtet. Selim könnte im Roman folglich auch als Referenz auf den durch die Tagebuchebene verbürgten ,realen' Selim gelesen werden, wenn man in einem strikten Verständnis des Referenzpakts argumentiert. Die doppelte Les- 
barkeit von Selim als Geschichte und ,reale' Person eröffnet aber die grundsätzliche Möglichkeit, Selim als Alter Ego Alexanders zu verstehen.

Die Tagebuchstelle findet ein Echo im ,Roman', das den Zwischenstatus von Selim als Person und Geschichte spiegelt: „Immer wenn er so über seinen halb gesehenen, halb ersponnenen „Winnetou der Rede“ gesprochen hatte, dachte Alexander über das Gesagte weiter nach und kam auf wieder Neues“ (NADOLNY 2005:342). Alexander definiert sich zum Zeitpunkt dieser Aussage über die Rhetorik, deren Verständnis er an Selim entwickelt. Seine eigene Identitätsentwicklung geht von dem „halb gesehenen“ Selim der Tagebuchebene aus. Ausformuliert und mit produktiver Kraft ausgestattet wird sie aber erst am „Winnetou der Rede“. Der intertextuelle Verweis macht deutlich, dass hier eine Diegesis das ,Eigentliche“ der Welt hervorbringt, so wie in den Romanen von Karl May ein Bild vom Fremden gezeichnet wird, das als ,Realität die Vorstellungswelt vieler Leser*innen geprägt hat. Selim wird als „Winnetou der Rede" ein Produkt von Alexanders Imagination, das letztlich referentielle Kraft gewinnt, indem es eine Wunschidentität materialisiert.

In diesem Sinn macht der Alexander der Tagebuchebene einen Unterschied von Hören und Schreiben aus, wenn er Selim im Gefängnis besucht: ,Endlich kann ich seine Geschichte weiterhören - und weiterschreiben" (NADOLNY 2005:240). Der Gedankenstrich verdeutlicht die Kluft zwischen der angehörten Geschichte des ,realen' Selim und dem Schreiben, das den Selim des ,Romans' in letzter Instanz auf Alexanders Tagebuch-Ich verpflichtet.

Alexanders Tagebuch-Ich reflektiert selbst, dass es keine biographisch getreue Erzählung über Selim abliefert: „Selim erzählt mir weiterhin sein Leben, obwohl er sich, so vermute ich, im Roman nicht wiederfindet" (NADOLNY 2005:253). Es ist Selim unmöglich, sich im Roman wiederzufinden, weil es keine Referenzbeziehung zwischen ihm und dem Selim des, Romans' zu geben scheint. Die enge Verbindung zur eigenen Person indiziert dahingegen ein Possessivpronomen: „Ich hätte mir denken können, daß ihn die Begegnung mit ,meinem 'Selim befremdet" (NADOLNY 2005:252), ,Mein[] Selim" ist eine Formulierung, die die Relation als Besitzverhältnis ausdrückt. Eine Binsenweisheit der Biographik, der Biograph verrate in seiner Erzählung mehr über sich selbst als über den Gegenstand, wird hier letztlich in ein Extrem ausgeweitet: der Biograph Alexander erschreibt sich selbst über den Fremden.

So ist auch Selims Rede letztlich kein biographisches Datum, sondern verweist auf die selbst-bildende Funktion, die sie für Alexander erfüllt. Ein Vergleich zweier Stellen unterstreicht diese Beobachtung. Die Figur Alexander beschreibt 
in beiden Passagen, wie die Figur Selim auf Türkisch, und somit für ihn unverständlich, erzählt:

Einer von den Glattgesichtigen beantwortete Fragen und erzählte etwas. Er gab sich dabei offenbar wenig Mühe, brummte mit gerunzelter Stirn, räusperte sich, unterbrach, um eine Zigarette anzuzünden - er wirkte fast unkonzentriert. [...] Vielleicht erzählte er Märchen, so waren ja die Orientalen. (NADOLNY 2005:48)

Damit nahm er aber gleich selbst das Wort, sprach schnell und sehr artikuliert, wurde dabei lauter, brüllte geradezu, stand auf. [...] Er beendete seine Rede mit einem wirksamen Wort und ließ eine Pause, damit Mesut antworten konnte. Der tat es nicht. [...] Alexander fragte nicht, worum es gegangen war. Er hatte für diese Szene bereits seine eigene Lesart und wollte sie beibehalten: Danton und Robespierre, um 1791. (NADOLNY 2005:219)

Diese Textstellen weisen eine vergleichbare Erzählsituation auf: Ein intern auf Alexander fokalisierter Erzähler liefert eine deskriptive Schilderung der Redeweise und schließt mit einer Wertung der „Szene[n]“. Durch die Detailschärfe wirkt jede Passage trotz des Präteritums zeitlich unmittelbar und der Redner gewinnt durch den Erzähler Gestalt. Diese Gestalt hängt maßgeblich von der Wahrnehmung der Figur ab, an der sich der Erzähler orientiert. Obwohl in beiden Fällen Selims Art des Redens beschrieben wird, unterscheiden sich die jeweils hervorgebrachten Gestalten deutlich voneinander. In der ersten Passage wirkt die Redeweise ,unkonzentriert" und zeichnet sich nicht durch rhetorische Brillanz aus, wie das Brummen, Räuspern und Abbrechen indizieren. In der zweiten Passage scheint die Redeweise hingegen das Muster für eine überzeugende rhetorische Leistung zu sein. Die Art der Darstellung korrespondiert mit dem kulturellen Deutungsmuster, das der nur in ihrer ,Materialität' wahrgenommenen Rede beigefügt wird, um ein Verstehen zu ermöglichen. Im ersten Fall ist es das Klischee vom orientalischen Märchenerzähler. Durch den veränderten Satzbau, der durch den Einschub des ,ja“ eher an mündlicher Kommunikation orientiert ist, erscheint diese Einordnung als hingeworfener Gemeinplatz, der von Desinteresse kündet. Anders verhält es sich in der späteren Begegnung: Selim wird in einer für Alexander zwingenden „Lesart“ zu Danton. Damit spiegelt sich im Bild des guten Rhetorikers Alexanders Ambition, selbst ein begabter Redner zu werden. Die vermeintliche Deskription erweist sich als Präskription, die Selim als das beschreibt, was Alexander selbst sein will. Das Erzählen bildet eine Gestalt, die jeweils abhängig von den aufgerufenen Topoi und Intentionen Alexanders ist und somit trotz der Namensidentität vom ,realen' Selim abgelöst ist. Die beschriebene Gestalt stellt eine Reflektor-Figur Alexanders dar.

Die ,Entdeckung' der selbst-produzierenden Kraft der Inszenierung beschreibt dabei insbesondere das kurze 122. Unterkapitel: „Erfinden statt Wissen hieß die 
Devise, sich selbst inszenieren!“ (NADOLNY 2005:311). Diese Spur greift das Tagebuch auf und gibt darüber Einblicke in Alexanders Schreibpraxis:

Seltsamerweise war mein Vorbild in ,Selbstdarstellung' nur Selim, niemals Mesut. Ich glaubte in Selim das Genie des Erzählens, der Lüge zu entdecken, studierte an ihm, wie man sich ein Gepräge gibt, um anderen die Zeit zu stehlen und dafür von ihnen geliebt zu werden. (NADOLNY 2005:318-319)

Die „Selbstdarstellung“ als performativer Akt kann auch als performatives Schreiben verstanden werden. Das Leben als Schreibakt ist für Alexander vom ,Vorbild' Selim bestimmt. An ihm studiert jener, ,wie man sich ein Gepräge gibt", also eine Form erhält. Selim erscheint hier für Alexander nur insofern von Interesse zu sein, als er sich über ihn performativ materialisieren kann.

In diesem Sinne überschneiden sich die Figuren Alexander und Selim auch immer wieder. Insbesondere die Rechts-Links-Händigkeit ist für diese Überkreuzungen exemplarisch. Während Selim als stolzer Linkshänder eingeführt wird (NADOLNY 2005:89), macht Alexander erst durch Selim die Entdeckung, ein „Rechts-Links-Händer“ zu sein (NADOLNY 2005:217). Ethel Matala de Mazza liest diese „pathognomischen Metaphern“ (MATALA DE MAZZA 1996:174) als Überschrift der jeweiligen Sprechweise der Figuren. Die Linkshändigkeit Selims interpretiert Matala de Mazza als „motorische Chiffre der Mühelosigkeit“ im Umgang mit Sprache (MATALA DE MAZZA 1996:176), während sich Alexanders theoretischer Zugriff darauf in seiner „Rechts-Links-Händigkeit“ spiegele (MATALA DE MAZZA 1996:177). Die jeweils grundsätzlich andere Veranlagung verbinde die Protagonisten zu Parallelfiguren (MATALA DE MAZZA 1996:172). Die Parallelität verdeutlicht die enge Beziehung zwischen den Figuren, kann jedoch auch als Konvergenz interpretiert werden. Alexander ist sich als eigentlicher Linkshänder, der als Kind umerzogen wurde, selbst fremd und erscheint erst im Linkshänder Selim in seiner ,eigentlichen“ Gestalt. Als „Rechts-LinksHänder" umschließt Alexander die räumliche Opposition und löst sie auf. Damit erscheint der Linkshänder Selim als integraler Bestandteil des „Rechts-LinksHänders" Alexander.

Seinen Figuren kommt der Erzähler des ,Romans', der mit dem autodiegetischen Erzähler des Tagebuchs identifiziert werden kann, auf erstaunlich ähnliche Weise nah; oder bleibt ihnen vice versa auf ähnliche Weise fern: „Er spricht dann eine Weile über die Merkwürdigkeit, daß ich über mich selbst in der dritten Person schreibe [...]" (NADOLNY 2005:473). So gibt das Tagebuch Ömer Bey wieder, einen ehemaligen türkischen Landrat, der Alexanders Manuskript liest. Als mögliche Annäherung an diese Besonderheit bietet sich ein Rekurs auf Lejeune an, der ,die Möglichkeit einer autobiographischen Erzählung ,in der 
dritten Person““ (LEJEUnE 1994:17) beschreibt: „Es gibt jedoch Autobiographien, in denen ein Teil des Textes den Protagonisten mit der dritten Person bezeichnet, während im restlichen Text Erzähler und Protagonist in der ersten Person verschmelzen [...]“" (LEJEUNE 1994:17). Alexander greift als Erzähler auf sich selbst, aber auch auf andere Figuren in der Form der aktorialen Mitsicht zu. Der autodiegetische Erzähler nutzt den Erzähler des ,Romans', um sich über die dritte Person den verschiedenen Figuren anzunähern und darüber Gestalt zu gewinnen. Das Selbst-Fremde erscheint erzähltheoretisch qua Wechsel der Erzählperspektive.

\section{Die Kunst der Lebensbeschreibung}

STEN NADOLNYS Roman partizipiert an der Kunst der Lebensbeschreibung. Der Text ist als Kunst Lebensbeschreibung. Selim überschreitet dabei die Autofiktion in zwei Aspekten: Erstens stellt er die Frage nach dem Subjekt der Selbstlebensbeschreibung im Inneren des Textes, so dass er den Diskursraum der Autobiographik betritt, ohne dabei von einem bios auszugehen. Dabei löst er sich zweitens vom Kriterium der Namensidentität ab, indem er den fiktionalen Raum nutzt, um die Fremdheit im Selbst darzustellen. Das „Nach-außenSetzen des ,Je est un autre““ erzeugt hier mehr als nur ein „Kippbild“, das einen „Zwischen den Aspekten , Welt ' (,Autorleben ') und Fiktion changierenden Lesemodus an[stößt]" (BRASS 2021:74). Das Tagebuch avisiert einen performativen Schreibakt, der vom Vorbild Selims ausgeht und Alexander über den ,Umweg‘ des Fremden eine Form verleiht. Das Fremde ist für Kristeva das eigene Unbewusste und das Erkennen von dessen Fremdheit führt zu dem Verlust einer Identität, die als essentiell gedacht wird: „Aber der Fremde, in sich selbst angesiedelt, hat dennoch kein Selbst“" (KRISTEVA 1990:18). Die ,Autofiktion“, die das Selbst aus einer dezentrierten Perspektive erschreibt, ist bedingt durch die Möglichkeit, am Platz des Anderen zu sein:

Mit dem anderen, mit dem Fremden leben konfrontiert uns mit der Frage, ob es möglich ist, ein anderer zu sein. Es geht nicht einfach - im humanistischen Sinn - um unsere Fähigkeit, den anderen zu akzeptieren, sondern darum, an seiner Stelle zu sein und das heißt, sich als anderer zu sich selbst zu denken und zu verhalten. (KRISTEVA 1990:23)

Im Fall von Alexander-Selim materialisiert sich diese Addition von Zentren in der Verdoppelung des erzählten Ichs in Selim und Alexander. Nimmt man den herausgearbeiteten Zusammenfall der hier bislang analytisch getrennten Erzählebenen ernst, so kann eine rekursive Lektüre im eigentlichen Sinne nicht mehr 
von einer extra- und intradiegetischen Ebene sprechen. Letztlich produziert der Text eine große Metalepse, wodurch eine Identifizierung von erzählendem und erzähltem Ich verunmöglicht wird. Nicht nur durch die bifokale Spaltung des Erzählobjekts, sondern auch durch die absolute De-Zentrierung der gesamten Erzählanlage wird das Subjekt,Alexander' demnach verfremdet. Indem Alexander seine Selbst-Fremdheit über Selim erschreibt, kann er ,sich als anderer zu sich selbst denken“ (KRISTEVA 1990:23). Weil simultan aber unklar bleiben muss, wo in einer solchen zur Metalepse tendierenden Erzählanlage eigentlich noch ein ,Zentrum' besteht, ist ,Alexander' nicht nur am Platz des Anderen, sondern immer schon am anderen Platz.

\section{Literatur}

Benne, Christian (2007): Was ist Autofiktion? Paul Nizons ,Erinnerte Gegenwart". In: PARry, Christoph / Platen, Edgar (eds.): Autobiographisches Schreiben in der deutschsprachigen Gegenwartsliteratur. Bd. 2: Grenzen der Fiktionalität und der Erinnerung. München, 293-304.

Bohnenkamp, Anne (1996): Von der Freiheit des Erzählens. Zur Poetik Sten Nadolnys. In: Bunzel, Wolfgang (ed.): Sten Nadolny. Eggingen, 17-39.

Bourdieu, PIERRE (1990): Die biographische Illusion. In: BIOS 3/1:75-81.

Brass, SEBASTIAN (2021): Nachdenken über Alice B. und Christa W. Autofiktionale Vexierbilder und Pseudonyme bei Gertrude Stein und Christa Wolf. In: Boy, AlinA / HÖVING, VANESSA/HolWECK, KATJA(eds.): Vexierbilder. Inszenierungen von Autor:innenschaft von 1800 bis zur Gegenwart. Paderborn, 71-95.

Bunzel, Wolfgang (1996): Sten Nadolnys Roman »Selim oder Die Gabe der Rede». Aufbau, Struktur, Erzählweise. In: DERS. (ed.): Sten Nadolny. Eggingen, 147-169.

Gumbrecht, Hans Ulrich (2007): Postmoderne. In: Müller, JAN-DiRK (ed.): Reallexikon der Deutschen Literaturwissenschaft. Neubearbeitung des Reallexikons der deutschen Literaturgeschichte. Bd. 3. Berlin / New York, 136-140.

GÜNTER, PETRA (1993): „Langsam komme ich zu mir und überlege, ob ich das aufschreiben soll “. Der Erzähler Sten Nadolny. In: Delabar, WAlter / Jung, Werner / Pergande, INGRID (eds.): Neue Generation - Neues Erzählen. Deutsche Prosa-Literatur der achtziger Jahre. Opladen, 35-45.

HoldenRIED, MichaEla (2000): Autobiographie. Stuttgart.

HoldenRIED, MichaEla (2017): „In eigener Sache [...] romanhaft lügen“? Wahrheitsreferenz, Fiktionalisierung und Fälschung in der Autobiographie. In: DEPKAT, Volker / Pyta, Wolfram (eds.): Autobiographie zwischen Text und Quelle. Geschichtsund Literaturwissenschaft im Gespräch I. Berlin, 57-75. 
Kristeva, Julia (1990): Fremde sind wir uns selbst. Aus dem Französischen von Xenia Rajewsky. Frankfurt a.M.

LECARME, JACQUES (1993): L'autofiction: un mauvais genre? In: DoubrovsKy, SERGE / LeCARme, JACQues / LeJeune, PhilipPe (eds.): Autofictions \& Cie. Nanterre, 227-249.

Lejeune, PhilipPe (1994): Der autobiographische Pakt. In: Ders.: Der autobiographische Pakt. Aus dem Französischen von Wolfram Bayer und Dieter Hornig. Frankfurt a.M., 13-55.

Matala de MAZZA, Ethel (1996): Schriftliche Wieder-Gabe. Zur Rhetorik der Kritik der Rhetorik in Nadolnys Roman „Selim oder Die Gabe der Rede“. In: BunZEL, WolfGANG (ed.): Sten Nadolny. Eggingen, 170-191.

Nadolny, Sten (2005): Selim oder Die Gabe der Rede. Roman. 11. Auflage. München.

WAGner-EgelhaAf, MARTina (2005): Autobiographie. 2., aktualisierte Auflage. Stuttgart.

Wagner-EgelhaAf, Martina (2006): Autofiktion oder: Autobiographie nach der Autobiographie. Goethe - Barthes - Özdamar. In: BreUER, UlRich / SANDBERG, BEATRICE (eds.): Autobiographisches Schreiben in der deutschsprachigen Gegenwartsliteratur. Bd. 1: Grenzen der Identität und der Fiktionalität. München, 353-368.

Wagner-EgelhaAf, Martina (2010): Zum Stand und zu den Perspektiven der Autobiographieforschung in der Literaturwissenschaft. In: BIOS 23, Heft 2: 188-200.

Wagner-EgelhaAf, Martina (2016): Autofiktion? Zur Brauchbarkeit einer Kategorie. In: Literaturstraße. Chinesisch-deutsche Zeitschrift für Sprach- und Literaturwissenschaft 17:15-21.

WAGNER-EgelHAAF, MARTINA (2017): Autobiographie als literaturwissenschaftiches Problem. In: DePKAT, Volker / PyTA, Wolfram (eds.): Autobiographie zwischen Text und Quelle. Geschichts- und Literaturwissenschaft im Gespräch I. Berlin, 43-57.

ZIPFEL, FRANK (2009): Autofiktion. Zwischen den Grenzen von Faktualität, Fiktionalität und Literarität? In: JANNIDIS, FOTIS / LAUER, GERHARD / WinKO, SimONE (eds.): Grenzen der Literatur. Zu Begriff und Phänomen des Literarischen. Berlin / New York, 285-315. 